The views expressed in this editorial are those of the author(s) and do not necessarily reflect the position of the Canadian Medical Association or its subsidiaries, the journal's editorial board or the Canadian College of Neuropsychopharmacology.

\title{
Adult neuroplasticity: A new "cure" for major depression?
}

\author{
Paul R. Albert, PhD
}

Neuroplasticity involves synaptic reorganization (Box 1) in response to environmental stressors or rewards and is thought to underlie our ability to adjust, learn and remember. ${ }^{7}$ However, in mental illness, it is thought that maladaptive plasticity occurs, resulting in persistence of the depressive symptoms, such as rumination, anhedonia and others. ${ }^{8}$ By harnessing corrective neuroplasticity (Box 2), it may be possible to reprogram the maladaptive behaviour and produce long-lasting remission. This editorial presents evidence that both pharmacological and brain stimulation antidepressant approaches may act by inducing corrective neuroplasticity to mediate remission. Increasingly, antidepressant effects on neuroplasticity are shown to correlate with behavioural improvement, both in humans ${ }^{18}$ and in animal models, ${ }^{19}$ raising the possibility that neuroplasticity could provide a new avenue for depression research. However, learning how to harness this capacity to enhance recovery remains a challenge.

\section{Measures of neuroplasticity in human depression}

In clinical studies, grey matter volume obtained from MRI provides an indirect indicator of neuronal density. Changes in grey matter volume are thought to reflect neuroplasticity (Box 1). But what is grey matter volume measuring? In major depressive disorder (MDD), it is clear that there is both a reduction in grey matter volume (especially in the subgenual anterior cingulate cortex ${ }^{20}$ and hippocampus ${ }^{21}$ ) and, from postmortem studies, a loss of both neurons and glia, particularly in those with chronic illness. ${ }^{22,23}$ Reduction in hippocampal volume in depression has been correlated with severity of memory impairments. ${ }^{24}$ In chronic social defeat mice, volume changes were region-specific and correlated inversely with social interactions. ${ }^{25}$ Reductions in hippocampal volume were also seen in a social depression model in female cynomolgus monkeys, ${ }^{26,27}$ which also showed reductions in serotonin $1 \mathrm{~A}\left(5-\mathrm{HT}_{1 \mathrm{~A}}\right)$ receptor levels. ${ }^{28}$ These grey matter volume reductions correlated with reduced cell numbers, predominantly of astroglial and granule cells, and with reduced cell

\section{Box 1: Defining neuroplasticity}

Neuroplasticity is a broad term, so how do we define it? At a systems level, it can encompass several discrete structural modifications that rewire the brain, ranging from early synaptic plasticity, either strengthening (long-term potentiation) or weakening (long-term depression) synaptic transmission; synapse formation or retraction; spinogenesis; synaptogenesis (also termed late synaptic plasticity); axonal sprouting; axon regeneration; dendrite growth and formation; ${ }^{1}$ and even neurogenesis. ${ }^{2,3}$ Synaptic plasticity is defined as a change in synaptic efficiency, but this sometimes includes formation of new synapses underlying late forms of synaptic plasticity. ${ }^{4}$ For the present discussion, neuroplasticity is as classically defined at a cellular level to denote structural change in neurons, ${ }^{5}$ thus excluding early synaptic plasticity and neurogenesis. In the context of brain imaging, the term neuroplasticity is applied to persistent or stable changes in brain functional activity (functional connectivity) or structure (grey matter volume) that are thought to be the result of cellular neuroplasticity. ${ }^{6}$

\section{Box 2: Triggers for adult neuroplasticity}

Initiation of neuroplasticity involves changes in neuronal activity. Neuronal activity triggers signalling pathways, including ERK $1 / 2^{9}$ and CREB signalling, ${ }^{10}$ leading to increased release of trophic factors, such as brain-derived neurotrophic factor ${ }^{4}$ or vascular endothelial growth factor, ${ }^{11}$ which in turn trigger transcriptional changes $^{12}$ that, if stimulation is persistent, result in structural changes, including formation of dendritic spines and recruitment of nerve terminals, resulting in new synaptic contacts. ${ }^{13}$ For example, exercise-induced behavioural improvement and neurogenesis are reduced in brain-derived neurotrophic factor (Met/Met) mutant mice. ${ }^{14}$ Some forms of plasticity (synapse reorganization) can occur in response to various stimuli, including drugs, exercise and enriched environment. ${ }^{15}$ However, different signalling pathways may trigger axonal sprouting or regeneration. Axonal regeneration in the adult central nervous system is fairly uncommon, but does occur in a subset of nonmyelinated axons, such as those of the serotonin system. ${ }^{16}$ Axonal sprouting is often triggered by neuronal activation following a brain injury, such as stroke, due to a loss of contralateral inhibition. ${ }^{17}$ It remains unclear whether axonal regeneration or sprouting play a role in the treatment of major depression, but these processes may occur in poststroke depression, where axonal projections are damaged.

Correspondence to: P. Albert, UOttawa Brain and Mind Research Institute, Ottawa Hospital Research Institute, 451 Smyth Rd, Ottawa, ON K1H-8M5; paul.albert@uottawa.ca 
and neuropil volumes mainly in the anterior hippocampus. ${ }^{29}$ In humans, the postmortem hippocampus of individuals with major depression showed similar reductions in both granule cell and astroglial cell numbers and reductions in cell and neuropil volumes. ${ }^{30}$ Reductions in hippocampal volume have also been associated with childhood maltreatment, ${ }^{31}$ a major risk factor for psychiatric disease and suicide. These studies showing reduced cell numbers and neuropil and grey matter volumes indicate an impairment of developmental or adult neuroplasticity in MDD. The causes of impaired neuroplasticity in MDD are unclear, but chronic increase in stress hormones replicates the reduction in hippocampal neuropil and is a likely culprit. ${ }^{32}$ Animal studies suggest that these stress-associated neuroplasticity processes may be prevented or reversed by antidepressant induction of neuroplasticity mediators such as neuritin; ${ }^{33}$ however, the mechanisms involved remain unclear (Box 2). Increasing evidence points to stress-induced microglial activation as a key contributor to synaptic remodelling, ${ }^{34-37}$ but how antidepressants or brain stimulation affect microglial responses in humans is unclear.

In order to assess functional neuroplasticity in humans, various brain stimulation approaches have been used, including paired associative stimulation (PAS) or transcranial magnetic stimulation (TMS). Depressed individuals show reduced PAS in the dorsolateral prefrontal cortex (DLPFC) compared with healthy individuals, suggesting reduced neuroplasticity. ${ }^{38}$ Selective serotonin reuptake inhibitors (SSRIs) affect neuroplasticity induced by transcranial direct current stimulation (tDCS), as detected by repetitive TMS (rTMS)-evoked motor cortex responses or PAS responses. ${ }^{39-41}$ Recently, magnetic seizure therapy has been shown to provoke changes in the excitability of the frontal cortex that correlate with a reduction in suicidal ideation..$^{42}$ It is hypothesized that by targeting areas that are most inactivated in MDD with chronic brain stimulation (e.g., rTMS), a more effective and sustained antidepressant response may be attained. ${ }^{43}$ Thus, sustained antidepressant responses may be driven by stimulation-induced synaptic reorganization.

\section{Serotonin and adult neuroplasticity}

Most antidepressants target monoamine reuptake, and SSRIs block the 5-HT transporter specifically. ${ }^{44}$ Recent postmortem studies show that in addition to reductions in grey matter volume, MDD is associated with reduced 5-HT innervation of the orbitofrontal cortex. ${ }^{45}$ The mechanisms underlying this deficiency are unclear. In this regard, impaired protein translation triggered by the cytokine tumour necrosis factor- $\alpha$ has been implicated in a reduction in 5-HT neurons and 5-HT-induced excitatory PFC responses associated with SSRI-resistant depression-like behaviour in mice. ${ }^{46,47}$ Given the role of 5-HT in SSRI action and the capacity of SSRIs to alter adult neuroplasticity in animal models via $5-\mathrm{HT}_{1 \mathrm{~A}}$ receptors, ${ }^{48,49}$ recent studies in humans have correlated grey matter volume with levels of 5-HT receptor binding detected using positron emission tomography or by measuring white matter density using diffusion tensor imaging (DTI). In healthy individuals, $5-\mathrm{HT}_{1 \mathrm{~A}}$ receptor binding was correlated with grey matter volume in several regions, including the hippocampus and $\mathrm{PFC},{ }^{50}$ while this correlation was lost in those with MDD, in whom a correlation with raphe (presynaptic) $5-\mathrm{HT}_{1 \mathrm{~A}}$ receptors emerged in restricted cortical areas. ${ }^{51}$ These correlations suggest an uncoupling of postsynaptic $5-\mathrm{HT}_{1 \mathrm{~A}}$ receptors and a predominant role of presynaptic $5-\mathrm{HT}_{1 \mathrm{~A}}$ autoreceptor-mediated inhibition of neuroplasticity in MDD. Interestingly, in MDD, the regional $5-\mathrm{HT}_{1 \mathrm{~A}}$-cortical thickness association correlated with the number of tracts from midbrain to specific cortical regions seen using DTI. ${ }^{52}$ This suggests that a strong interaction of 5-HT projections with the default mode network may drive increased rumination associated with MDD. ${ }^{53}$ Taken together, these and other studies implicate the 5-HT system in changes in neuroplasticity occurring in major depression.

Recently, a receptor-mediated mechanism has been identified that could underlie SSRI-associated cortical neuroplasticity, which involves $5-\mathrm{HT}_{7}$ receptor signalling to activate metalloproteinases to mediate synapse formation. ${ }^{54}$ In the mouse chronic social defeat model, alterations in excitatory/ inhibitory input to 5-HT neurons and of synapse size and number in the hippocampus were reversed by chronic deep brain stimulation in parallel with its antidepressant actions. ${ }^{55}$ These results in mice suggest that activation of 5-HT neurons can trigger neuroplasticity of the 5-HT system. In this regard, an increase in cortical thickness seen upon acute (1 wk) SSRI treatment was associated with increased response (at $8 \mathrm{wk}$ ) in MDD. ${ }^{56}$ In humans, successful treatment with rTMS has been shown to increase hippocampal volume and is associated with cognitive improvement in depressed patients. ${ }^{57}$ Interestingly, the combination of SSRI and anodal tDCS stimulation enhanced memory function, while either treatment alone was ineffective. ${ }^{58}$ Thus, combining brain stimulation with SSRI treatment to increase 5-HT neurotransmission could be a promising approach for treatmentresistant depression.

\section{Ketamine and adult neuroplasticity}

Acute treatment with low-dose ketamine has been shown to produce rapid (within $1 \mathrm{~h}$ ) improvements in treatment-resistant $\mathrm{MDD},{ }^{59,60}$ particularly in reducing suicidality. ${ }^{61}$ Ketamine acts like brain stimulation to increase glutamatergic activity (glutamate surge) inducing brain-derived neurotrophic factor release to trigger the formation of new dendritic spines. ${ }^{62}$ However, owing to risk of psychosis, it is used only acutely, but recent trials suggest that repeated ketamine administration may be effective and safe for treatment-resistant depression. ${ }^{63-65}$ It also remains unclear to what extent ketamine's antidepressant actions in humans depend on neuroplasticity. In rodents, inhibitors of the mammalian target of rapamycin that reduce dendritic protein synthesis prevent ketamineinduced neuroplasticity and behavioural improvement, ${ }^{66}$ suggesting a role for neuroplasticity, although additional mechanisms are implicated in ketamine action. ${ }^{67}$ Whether ketamine could "kick-start" the response to SSRI treatment has not been studied extensively, although initial results suggest improved response and remission rates compared with placebo. ${ }^{68}$ 


\section{Conclusion}

Evidence from animal models of depression and increasing, but indirect evidence from human studies suggest that neuroplasticity is impaired in MDD. Both in humans and in animal models, treatment with brain stimulation (ketamine, DBS, or other methods) induces regional increases in grey matter volume that are associated with antidepressant response. These brain volume changes involve structural neuroplasticity mechanisms, such as dendritic spinogenesis, synaptic reorganization, or axonal sprouting or regrowth. In humans, SSRIs, brain stimulation or their combination may enhance adult neuroplasticity to mediate recovery in MDD. However, it remains to be elucidated what is the exact role of impaired neuroplasticity in human depression and whether triggering neuroplasticity could accelerate or potentiate antidepressant responses.

\section{References}

1. Warraich Z, Kleim JA. Neural plasticity: the biological substrate for neurorehabilitation. PM R 2010;2(Suppl 2):S208-19.

2. Fuchs E, Flügge G. Adult neuroplasticity: more than 40 years of research. Neural Plast 2014;2014:541870.

3. Toda T, Gage FH. Review: adult neurogenesis contributes to hippocampal plasticity. Cell Tissue Res 2018;373:693-709.

4. Sweatt JD. Neural plasticity and behavior - sixty years of conceptual advances. J Neurochem 2016;139(Suppl 2):179-99.

5. Berlucchi G, Buchtel HA. Neuronal plasticity: historical roots and evolution of meaning. Exp Brain Res 2009;192:307-19.

6. Zatorre RJ, Fields RD, Johansen-Berg H. Plasticity in gray and white: neuroimaging changes in brain structure during learning. Nat Neurosci 2012;15:528-36.

7. Cameron HA, Schoenfeld TJ. Behavioral and structural adaptations to stress. Front Neuroendocrinol 2018;49:106-13.

8. McEwen BS, Eiland L, Hunter RG, et al. Stress and anxiety: structural plasticity and epigenetic regulation as a consequence of stress. Neuropharmacology 2012;62:3-12.

9. Krapivinsky G, Krapivinsky L, Manasian Y, et al. The NMDA receptor is coupled to the ERK pathway by a direct interaction between NR2B and RasGRF1. Neuron 2003;40:775-84.

10. D'Sa C, Duman RS. Antidepressants and neuroplasticity. Bipolar Disord 2002;4:183-94.

11. Warner-Schmidt JL, Duman R. VEGF as a potential target for therapeutic intervention in depression. Curr Opin Pharmacol 2008;8:14-9.

12. McClung CA, et al. Neuroplasticity mediated by altered gene expression. Neuropsychopharmacology 2008;33:3-17.

13. Pittenger C, Duman RS. Stress, depression, and neuroplasticity: a convergence of mechanisms. Neuropsychopharmacology 2008;33:88-109.

14. Ieraci A, Madaio AI, Mallei A, et al. Brain-derived neurotrophic factor Val66Met human polymorphism impairs the beneficial exerciseinduced neurobiological changes in mice. Neuropsychopharmacology 2016:41:3070-9.

15. Kobilo T, Liu QR, Gandhi K, et al. Running is the neurogenic and neurotrophic stimulus in environmental enrichment. Learn Mem 2011;18:605-9.

16. Jin Y, Dougherty SE, Wood K et al. Regrowth of serotonin axons in the adult mouse brain following injury. Neuron 2016;91:748-62.

17. Carmichael ST. Plasticity of cortical projections after stroke. Neuroscientist 2003;9:64-75.

18. Kraus C, Castrén E, Kasper S, et al. Serotonin and neuroplasticity - links between molecular, functional and structural pathophysiology in depression. Neurosci Biobehav Rev 2017;77:317-26.

19. Neto FL, Borges G, Torres-Sanchez D et al. Neurotrophins role in depression neurobiology: a review of basic and clinical evidence. Curr Neuropharmacol 2011;9:530-52.

20. Savitz JB, Drevets WC. Imaging phenotypes of major depressive disorder: genetic correlates. Neuroscience 2009;164:300-30.

21. Campbell S, Marriott M, Nahmias C, et al. Lower hippocampal volume in patients suffering from depression: a meta-analysis. Am J Psychiatry 2004;161:598-607.
22. Rajkowska G, Miguel-Hidalgo JJ, Wei J, et al. Morphometric evidence for neuronal and glial prefrontal cell pathology in major depression. Biol Psychiatry 1999;45:1085-98.

23. Cobb JA, Simpson J, Mahajan GJ, et al. Hippocampal volume and total cell numbers in major depressive disorder. J Psychiatr Res 2013; 47:299-306.

24. MacQueen GM, Campbell S, McEwen B et al. Course of illness, hippocampal function, and hippocampal volume in major depression. Proc Natl Acad Sci U S A 2003;100:1387-92.

25. Anacker C, Scholz J, O'Donnell KJ, et al. Neuroanatomic differences associated with stress susceptibility and resilience. Biol Psychiatry 2016;79:840-9.

26. Willard SL, Daunais JB, Cline JM et al. Hippocampal volume in postmenopausal cynomolgus macaques with behavioral depression. Menopause 2011;18:582-6.

27. Willard SL, Friedman DP, Henkel CK, et al. Anterior hippocampal volume is reduced in behaviorally depressed female cynomolgus macaques. Psychoneuroendocrinology 2009;34:1469-75.

28. Shively CA, Friedman DP, Gage HD, et al. Behavioral depression and positron emission tomography-determined serotonin $1 \mathrm{~A}$ receptor binding potential in cynomolgus monkeys. Arch Gen Psychiatry 2006; 63:396-403.

29. Willard SL, Riddle DR, Forbes ME, et al. Cell number and neuropil alterations in subregions of the anterior hippocampus in a female monkey model of depression. Biol Psychiatry 2013;74:890-7.

30. Stockmeier CA, Mahajan GJ, Konick LC, et al. Cellular changes in the postmortem hippocampus in major depression. Biol Psychiatry 2004;56:640-50.

31. Teicher MH, Anderson CM, Polcari A. Childhood maltreatment is associated with reduced volume in the hippocampal subfields CA3, dentate gyrus, and subiculum. Proc Natl Acad Sci U S A 2012;109: E563-72.

32. McEwen BS. Stress and hippocampal plasticity. Annu Rev Neurosci 1999;22:105-22.

33. Son $\mathrm{H}$, Banasr M, Choi M, et al. Neuritin produces antidepressant actions and blocks the neuronal and behavioral deficits caused by chronic stress. Proc Natl Acad Sci U S A 2012;109:11378-83.

34. Wohleb ES. Neuron-microglia interactions in mental health disorders: "for better, and for worse". Front Immunol 2016;7:544.

35. Wohleb ES, Franklin T, Iwata M, et al. Integrating neuroimmune systems in the neurobiology of depression. Nat Rev Neurosci 2016; 17:497-511.

36. Mechawar N, Savitz J et al. Neuropathology of mood disorders: Do we see the stigmata of inflammation? Transl Psychiatry 2016;6:e946.

37. Singhal G, Baune BT. Microglia: an interface between the loss of neuroplasticity and depression. Front Cell Neurosci 2017;11:270.

38. Noda Y, Zomorrodi R, Vila-Rodriguez F ,et al. Impaired neuroplasticity in the prefrontal cortex in depression indexed through paired associative stimulation. Depress Anxiety 2018;35:448-56.

39. Nitsche MA, Kuo MF, Karrasch R, et al. Serotonin affects transcranial direct current-induced neuroplasticity in humans. Biol Psychiatry 2009:66:503-8.

40. Batsikadze G, Paulus W, Kuo MF, et al. Effect of serotonin on paired associative stimulation-induced plasticity in the human motor cortex. Neuropsychopharmacology 2013;38:2260-7.

41. Kuo HI, Paulus W, Batsikadze G, et al. Chronic enhancement of serotonin facilitates excitatory transcranial direct current stimulationinduced neuroplasticity. Neuropsychopharmacology 2016;41:1223-30.

42. Sun Y, Blumberger DM, Mulsant BH, et al. Magnetic seizure therapy reduces suicidal ideation and produces neuroplasticity in treatment-resistant depression. Transl Psychiatry 2018;8:253.

43. Dubin MJ, Liston C, Avissar MA, et al. Network-guided transcranial magnetic stimulation for depression. Curr Behav Neurosci Rep 2017; 4:70-7.

44. Wong DT, Perry KW, Bymaster FP. Case history: the discovery of fluoxetine hydrochloride (Prozac). Nat Rev Drug Discov 2005;4:764-74.

45. Rajkowska G, Mahajan G, Legutko B, et al. Length of axons expressing the serotonin transporter in orbitofrontal cortex is lower with age in depression. Neuroscience 2017;359:30-9.

46. Aguilar-Valles A, Haji N, De Gregorio D ,et al. Translational control of depression-like behavior via phosphorylation of eukaryotic translation initiation factor 4E. Nat Commun 2018;9:2459.

47. Amorim IS, Kedia S, Kouloulia S, et al. Loss of eIF4E phosphorylation engenders depression-like behaviors via selective mRNA translation. J Neurosci 2018;38:2118-33. 
48. Maya Vetencourt JF, Sale A, Viegi A, et al. The antidepressant fluoxetine restores plasticity in the adult visual cortex. Science 2008;320:385-8.

49. Maya Vetencourt JF, Tiraboschi E, Spolidoro M, et al. Serotonin triggers a transient epigenetic mechanism that reinstates adult visual cortex plasticity in rats. Eur I Neurosci 2011;33:49-57.

50. Kraus C, Hahn A, Savli M, et al. Serotonin-1A receptor binding is positively associated with gray matter volume - a multimodal neuroimaging study combining PET and structural MRI. Neuroimage 2012;63:1091-8.

51. Zanderigo F, Pantazatos S, Rubin-Falcone $\mathrm{H}$, et al. In vivo relationship between serotonin 1A receptor binding and gray matter volume in the healthy brain and in major depressive disorder. Brain Struct Funct 2018;223:2609-25.

52. Pillai RLI, Malhotra A, Rupert DD, et al. Relations between cortical thickness, serotonin $1 \mathrm{~A}$ receptor binding, and structural connectivity: a multimodal imaging study. Hum Brain Mapp 2018;39:1043-55.

53. Dutta A, McKie S, Deakin JF. et al. Resting state networks in major depressive disorder. Psychiatry Res 2014;224:139-51.

54. Bijata M, Labus J, Guseva D et al. Synaptic remodeling depends on signaling between serotonin receptors and the extracellular matrix. Cell Reports 2017;19:1767-82.

55. Veerakumar A, Challis C, Gupta P et al. Antidepressant-like effects of cortical deep brain stimulation coincide with pro-neuroplastic adaptations of serotonin systems. Biol Psychiatry 2014;76:203-12.

56. Bartlett EA, DeLorenzo C, Sharma P, et al. Pretreatment and earlytreatment cortical thickness is associated with SSRI treatment response in major depressive disorder. Neuropsychopharmacology 2018;43:2221-30.

57. Noda Y, Zomorrodi R, Daskalakis ZJ, et al. Enhanced theta-gamma coupling associated with hippocampal volume increase following high-frequency left prefrontal repetitive transcranial magnetic stimulation in patients with major depression. Int J Psychophysiol 2018;133:169-74.

58. Prehn K, Stengl H, Grittner U, et al. Effects of anodal transcranial direct current stimulation and serotonergic enhancement on memory performance in young and older adults. Neuropsychopharmacology 2017;42:551-61.

59. Newport DJ, Carpenter LL, McDonald WM et al. Ketamine and other NMDA antagonists: early clinical trials and possible mechanisms in depression. Am J Psychiatry 2015;172:950-66.

60. Zarate CA Jr, Singh JB, Carlson PJ, et al. A randomized trial of an $\mathrm{N}$-methyl-D-aspartate antagonist in treatment-resistant major depression. Arch Gen Psychiatry 2006;63:856-64.

61. Grunebaum MF, Galfalvy $\mathrm{HC}, \mathrm{Choo} \mathrm{TH}$, et al. Ketamine for rapid reduction of suicidal thoughts in major depression: a midazolamcontrolled randomized clinical trial. Am I Psychiatry 2018;175:327-35.

62. Abdallah CG, Sanacora G, Duman RS, et al. Ketamine and rapidacting antidepressants: a window into a new neurobiology for mood disorder therapeutics. Annu Rev Med 2015;66:509-23.

63. Domany Y, Bleich-Cohen M, Tarrasch R, et al. Repeated oral ketamine for out-patient treatment of resistant depression: randomised, double-blind, placebo-controlled, proof-of-concept study. Br J Psychiatry 2019;214:20-6.

64. Ionescu DF, Bentley KH, Eikermann M, et al. Repeat-dose ketamine augmentation for treatment-resistant depression with chronic suicidal ideation: a randomized, double blind, placebo controlled trial. I Affect Disord 2019;243:516-24.

65. Phillips JL, Norris S, Talbot J, et al. Single, repeated, and maintenance ketamine Infusions for treatment-resistant depression: a randomized controlled trial. Am J Psychiatry 2019:appiajp201818070834 doi: 10.1176/appi.ajp.2018.18070834. [Epub ahead of print].

66. Li N, Lee B, Liu RJ, et al. mTOR-dependent synapse formation underlies the rapid antidepressant effects of NMDA antagonists. Science 2010;329:959-64.

67. Zanos P, Gould TD. Mechanisms of ketamine action as an antidepressant. Mol Psychiatry 2018;23:801-11.

68. Hu YD, Xiang YT, Fang JX, et al. Single i.v. ketamine augmentation of newly initiated escitalopram for major depression: results from a randomized, placebo-controlled 4-week study. Psychol Med 2016;46: 623-35.

\section{Journal of Psychiatry e) Neuroscience}

\section{Call for submissions}

Have expertise treating patients with psychiatric disorders? Share it with clinicians in a Psychopharmacology for the Clinician column. Columns are 650 words and include a clinical vignette showcasing a topic of interest. Cases should have a level of complexity or novelty that will help clinicians make treatment decisions in situations that are not routine, or where new evidence is available but not widely known.

\begin{abstract}
Why write for JPN?
- JPN is the highest ranking open access journal in biological psychiatry

- Psychopharmacology for the Clinician columns are the most downloaded feature of JPN and archives are available indefinitely on jpn.ca and in PubMed Central
\end{abstract}

